\title{
Simulation Research on Application of Azimuth Electromagnetic Wave in Coal Seam Detection
}

\author{
CHEN Gang ${ }^{1,2}$, CHEN Long ${ }^{2}$, Fan Yiren ${ }^{1}$, Li Quanxin ${ }^{2}$ \\ ${ }^{1}$ School of Geosciences, China University of Petroleum (East China), Qingdao, 266580, China \\ ${ }^{2}$ CCTEG XI'AN Research Institute, Xi'an, Shaanxi, 710077, China
}

\begin{abstract}
Aiming at the problems of poor detection accuracy and low efficiency on the roof and floor interface in horizontal well drilling in coal mine, the design idea of explosion-proof azimuth-while-drilling electromagnetic wave logging instrument in coal mine was put forward. In order to suit the working environment of underground coal seam, the change law of the influence of instrument parameters such as source distance and coil installation angle on the signal response was obtained through forward simulation. Through the simulation research on the response of azimuth-while-drilling electromagnetic wave logging instrument, it provided a basis for selection of parameter optimization design of the instruments applicable to the underground coal mine, and the change law of the response of coal rock roof and floor interface was mastered. The application of this instrument will improve the detection accuracy and efficiency on the roof and floor.
\end{abstract}

\section{Introduction}

Due to the limitation of working environment and conditions, currently there is no relevant azimuth electromagnetic wave detection instrument in gas extraction from underground coal mines. With the continuously deepening of coalbed methane exploration and development, the complexity of geological conditions and the limitation of seismic data are increasing. Coalbed methane drilling and mining in underground coal mine often encounters unclear problems such as unpredictable changes in the thickness of the coal seam, lateral fluctuations and the conditions of mudstone interlayers, changeable structure, etc. which makes it quite difficult to detect coal seams ${ }^{[1-3]}$. This has led to the rapid development of measurement-while-drilling equipment, and geological guidance can be realized by means of measurement-while-drilling equipment, which can effectively improve the accuracy and effectiveness of detection. In order to increase the per-well production of coalbed methane, a horizontal pinnate branching well drilling and production technology was proposed, which could effectively improve the output and production efficiency of coalbed methane. However, how to avoid escaping from the coal seam and keep the wellbore track along the coal seam, and simultaneously detect the location of the roof and floor interface as well as the distance and direction to the interface, are the practical problems faced in the development of coalbed methane ${ }^{[4-}$ 7].

In view of the above, this paper simulated and studied the detection performance of the explosion-proof azimuth- while-drilling electromagnetic wave instrument under different parameters on the roof and floor interface in coal seam, and used physical simulation experiments to optimize the coil system structure scheme of the instrument. By tilting the transmitting coil, the azimuthwhile-drilling resistivity logging instrument was equipped with azimuth detection capability, and the received directional attenuation signal could clearly predict and indicate the existence of the layer interface.

\section{Selection of source distance and response simulation}

When the transmitting frequency and coil inclination are determined, the source distance of the instrument determines its radial detection depth. In order to ensure that the design range of the source distance is suitable for high-resistance coal seams, the variation curve of the amplitude of the received induction signal with the source distance was obtained by simulation, shown in Fig. 1. 


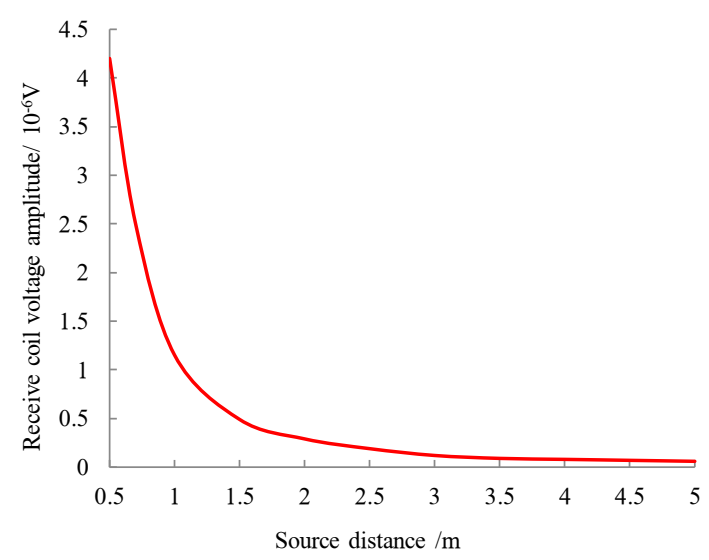

Fig. 1 The relationship between the amplitude of the induction signal and the variation of the coil source distance

Due to the narrow working space of the coal mine roadway, the length of drill pipe used is usually no more than $3 \mathrm{~m}$, so the source distance selection must not exceed $2.5 \mathrm{~m}$. At the same time, in order to improve the signal-tonoise ratio of the attenuation signal of the receiver coil and meet the measurement accuracy of the instrument, the amplitude of the voltage of the receiver coil must be greater than $0.2 \times 10^{-6} \mathrm{~V}$. Fig. 2 shows the response changes of the amplitude ratio signal when the source distance is $2.2 \mathrm{~m}$ and $1.8 \mathrm{~m}$ respectively, and the corresponding transmission frequency is $100 \mathrm{kHz}$, the coil inclination angle is $45^{\circ}$, the coal seam resistivity is 200 $\Omega \cdot \mathrm{m}$, and the roof and floor resistivity are $10 \Omega \cdot \mathrm{m}$. It can be seen from Fig. 2 that the larger the source distance is, the stronger the directional reflection signal at the interface is, the larger the peak value is, and the more significant the boundary effect formed when crossing an interface is. The directional attenuation signal is positive when the instrument enters the high-resistance coal seam from the low-resistance layer on the roof, while the directional attenuation signal is negative when the instrument enters the low-resistance layer on the floor from the coal seam.

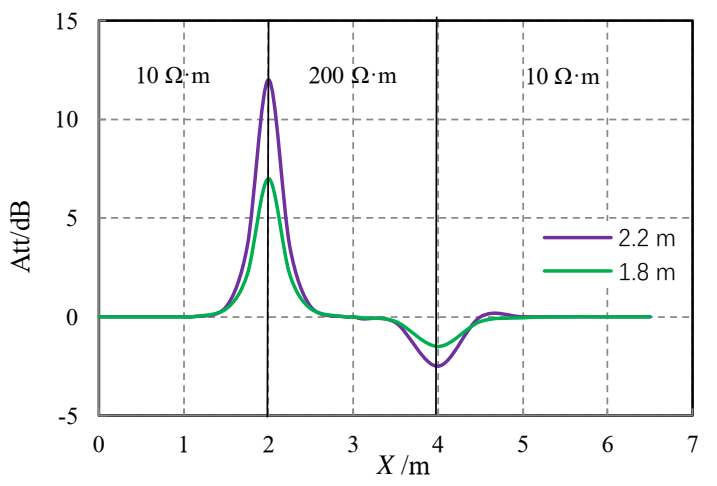

Fig. 2 Response of boundary effect with the variation of instrument source distance

\section{Response simulation of the installation angle of the receiver coil}

The placement angle of the receiver coil system directly affects the strength of the signal response of the reservoir limit test, and a relatively ideal boundary response can be obtained by changing the installation angle of the receiver antenna. When the installation angles of the receiver antenna were $30^{\circ}, 45^{\circ}$, and $60^{\circ}$, the Green function was used to calculate the received signal of the coil.

The magnetic field intensity at the source point is shown in the matrix of equation (1) ${ }^{[8-11]}$, and the response curve of the influence of coal seam boundary varying with the coil installation angle was calculated, shown in Fig.3.

Where the components of the magnetic field strength produced by the unit magnetic dipole source in the direction $\mathrm{x}$ are $G_{x x}^{\mathrm{HM}}, G_{x y}^{\mathrm{HM}}$ and $G_{x z}^{\mathrm{HM}}$, respectively; the components of the magnetic field strength produced by the unit magnetic dipole source in the direction y are $G_{y x}^{\mathrm{HM}}$, $G_{y y}^{\mathrm{HM}}$ and $G_{y z}^{\mathrm{HM}}$, respectively; the components of the magnetic field strength produced by the unit magnetic dipole source in the direction z are $G_{z x}^{\mathrm{HM}}, G_{z y}^{\mathrm{HM}}$ and $G_{z z}^{\mathrm{HM}}$, respectively.

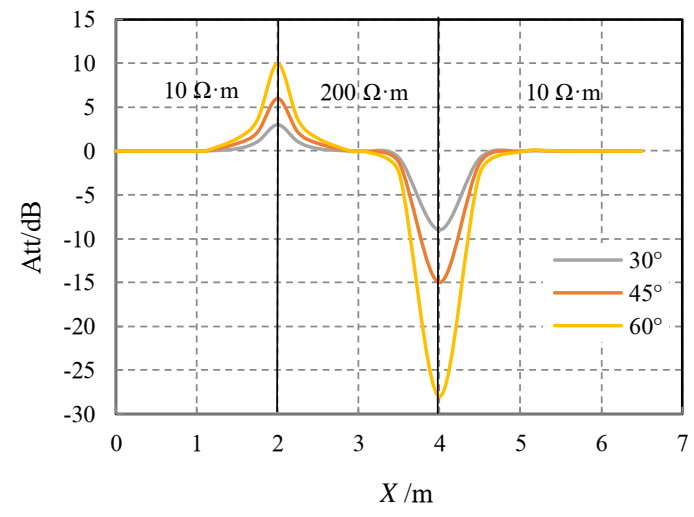

Fig.3 The response curve of boundary effect changing with the installation angle of the coil

As can be seen from Fig. 3, with a source distance of $2.2 \mathrm{~m}$, a transmission frequency of $100 \mathrm{kHz}$, a coil inclination angle of $45^{\circ}$, a coal seam resistivity of 200 $\Omega \cdot \mathrm{m}$, and the roof and floor resistivity of $10 \Omega \cdot \mathrm{m}$, the response of the directional amplitude attenuation signal becomes more obvious when it approaches the interface as the tilt angle of the receiver coil increases, and the larger the tilt angle, the larger the boundary response peak. However, as the inclination angle of the receiver coil increases, the induced electromotive force of the signal received by the coil weakens, which increases the signal error, so it is not that the larger the coil inclination angle is, the better. Generally, the inclination angle of $45^{\circ}$ is chosen as a compromise, which not only satisfies the sensitivity of the received signal, but also considers the strength of the signal.

\section{Response simulation of coal seam strike and boundary distance}

The azimuth-while-drilling electromagnetic wave resistivity instrument has great difference in receiving response signals under different coal seam strike. In order to study its corresponding characteristics, the instrument responds to the different interface dip of coal seam were 
stimulated under the condition that the source distance, transmitting frequency and coil inclination angle were determined. The simulation results are shown in Fig. 4. As can be see, with the increase of the inclination angle of the coal seam interface, the received signal peak value of interface reflection enlarges and the detection sensitivity increases.

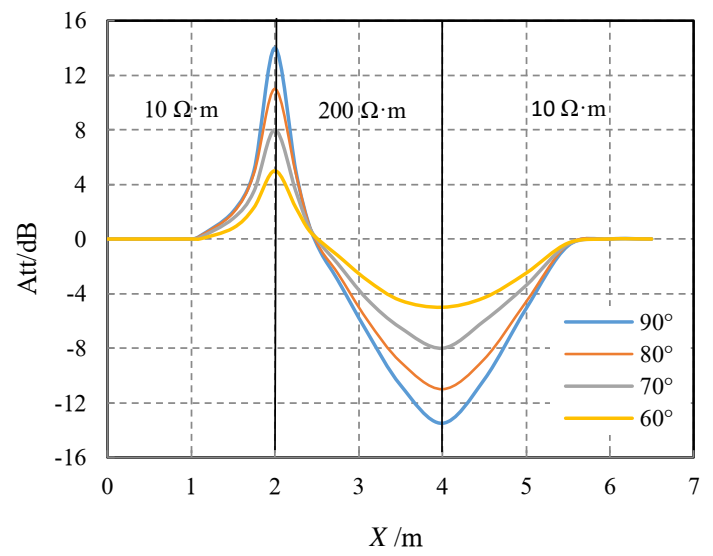

Fig. 4 Directional response of the instrument with different relative inclination angle of the coal seam interface

Generally, the resistivity of coal seam is quite different from that of roof and floor, and the directional attenuation signal of the receiver coil can clearly indicate the interface of the coal seam. By using the directional signal amplitude, the distance of layer interface can be calculated by inversion, so that the drill can keep working along the coal seam. Fig. 5 shows the response of the voltage amplitude of the receiver coil with the distance from the instrument to the boundary of the coal seam, as well as the response curve under different resistivity of the roof and floor. It can be seen from Fig. 5 that the contrast between voltage amplitude and the floor resistivity changes obviously, and the resistivity of the coal seam where the instrument is located is $200 \Omega \cdot \mathrm{m}$, and the resistivity of the roof and floor are $2 \Omega \cdot \mathrm{m}, 5 \Omega \cdot \mathrm{m}, 10 \Omega \cdot \mathrm{m}, 20 \Omega \cdot \mathrm{m}$. The smaller the simulated resistivity of roof and floor, the greater the response of voltage amplitude. When the roof and floor resistivity is determined, the voltage amplitude of the receiver coil varies monotonously with the distance from the instrument to the interface. The closer the instrument is to the boundary, the stronger the response of the voltage amplitude of the receiver coil.

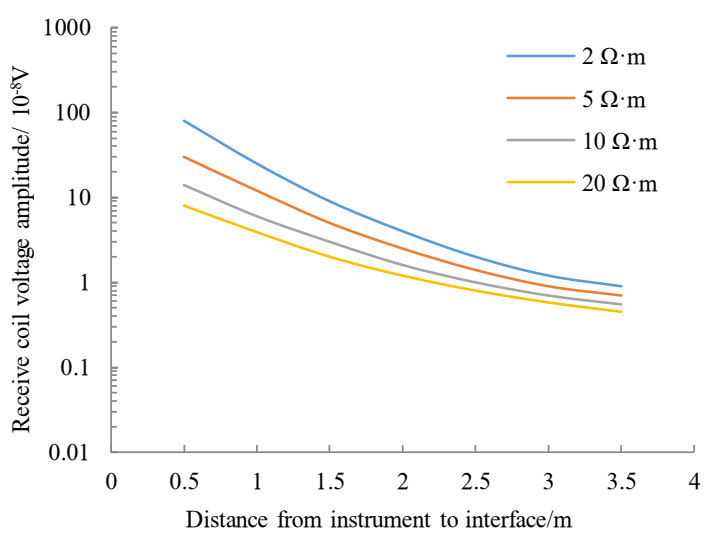

Fig. 5 The relationship between the voltage amplitude response and the distance from the instrument to the coal seam boundary, and the influence of the changes in resistivity of the roof and floor

\section{Conclusions}

By simulating the effects of the design parameters of the instrument, the change law of the response of the electromagnetic wave signal in the geological model were obtained. The research results showed that:

Through research on the detection method for coal seam boundary and the corresponding instrument design, the relationship between the structural parameters of the instrument and the installation angle of the coil and the response signal was obtained by simulation. The installation angle of the coil should be set to $45^{\circ}$, which was a compromise not only to satisfy the sensitivity of the received signal but also to take the strength of the signal into account. For the practical application of different measurement targets and constraints, the radial detection depth and longitudinal resolution of the required instrument could be designed, and a reasonable transmission frequency could improve the detection performance of the instrument. According to the calculation, the transmission frequency should be $100 \mathrm{kHz}$ $\sim 1 \mathrm{MHz}$.

b. Except the influence factors of the instrument's parameters, the peak value of the attenuation signal was also affected by the distance from the instrument to the stratigraphical boundary and the contrast of the formation resistivity. The closer the distance to the layer interface, the greater the contrast of the formation resistivity, and the larger the peak value of the response signal. In addition, the thicker the coal seam and the larger the relative inclination of the coal seam, the larger the peak value of the response signal and the higher the detection sensitivity. By judging the change of the voltage value and the sign of the receiver coil, it could not only indicate the position where the instrument crossed the interface of the coal seam, but also could determine whether it crossed from the coal seam to the roof and floor or from the roof and floor to the coal seam .

\section{Acknowledgments}

This paper was supported by Major National Science and 
Technology Special Tasks in the 13th Five-Year Plan (2016ZX05045-003-001) and Technology Innovation Program of CCTEG XI'AN Research Institute (2017XAYMS01).

\section{References}

1. Li Mingchao, Liang shengzheng, Zhao kejing. Coalbed methane and its exploration and development [M]. Beijing: Geological Publishing House, 1996

2. JIN Dewu, LIU Yingfeng, CHENG Jianyuan. New progress in prevention and control technology of major water inrush disasters in coal mines[J]. Coal Science and Technology. Coal science and technology, 2013, 41(1): 25-29.

3. MEN Xiangyong, HAN Zheng, GAO Baishui. Present situation and development suggestions of CBM exploration and development in China[J]. China Mining, 2017, 26(s2): 1-4.

4. SHI Zhijun, DONG Shuning, YAO Ningping, et al. Directional drilling technology and equipment for near-horizontal MWD in coal mines[J]. Coal Science and Technology, 2013, 41(3): 1-6.

5. JIANG Tingting, YANG Xiujuan, YAN Xiangzhen, et al. Influence of branch parameters on productivity of plume horizontal wells of coalbed methane[J]. Journal of China Coal Society, 2013, 38(4): 617-623.
6. ZHANG Hong, HE Aiguo. Structural optimization of pinnate branch horizontal wells[J]. Coalbed Methane of China, 2011, 21 (5): 26-29.

7. SHI Zhijun, YAO Ningping, YE Genfei. Construction technology and equipment of gas drainage borehole in coal mine[J]. Coal Science and Technology, 2009, 21(7): 1-4.

8. WAN Yong, ZHANG Xiaobin, NI Weining, et al. Research on the design of coil system for azimuthal propagation resistivity $\mathrm{LWD}[\mathrm{J}]$. Journal of Electronic Measurement and Instrumentation. 2017, 31(1): 99105.

9. ZHU Weixu, YUAN Zhiyong, TONG Qianqian. Coil magnetic field characteristics analysis and coil spatial pose calculation in magnetic haptic feedback[J]. Journal of Electronic Measurement and Instrumentation, 2016, 30(4): 590-597.

10. YANG Jinzhou, WEI Baojun, LIN Nan. Basic theory of electromagnetic wave resistivity measurement while drilling tool with tilted antennas and its application for Geo-steering[J]. Journal of China University of Petroleum, 2009, 33(1): 44-49.

11. CHEN Gang, FAN Yiren, LI Quanxin. A study of Coalbed Methane (CBM) reservoir boundary detections based on azimuth electromagnetic waves[J]. Journal of Petroleum Science and Engineering, 2019, 179: 432-443. 